\title{
Childhood nephrotic syndrome: steroid sensitive, steroid resistant and beyond
}

\author{
Asiri S Abeyagunawardena ${ }^{1}$ \\ Sri Lanka Journal of Child Health, 2009: 38: 115-120
}

(Key words; Childhood nephrotic syndrome, steroid sensitive, steroid resistant)

Nephrotic syndrome (NS) is the commonest glomerular disorder in childhood. It is a chronic distressing disorder characterised by heavy proteinuria, hypoproteinaemia, oedema and hyperlipidaemia. In the pre-antibiotic era children with NS often died, usually from overwhelming infections arising as a result of the immunosuppression which is an inherent feature of the disease and, poor nutrition. The importance of the immune system in the pathogenesis of childhood NS was first suggested by Shalhoub in 1974. However, even many decades before this, it was known that after an attack of measles, NS could enter long term remission.

In 1978 an interesting case report was published on a 10 year old boy who developed nephrotic syndrome more than 60 years ago ${ }^{1}$. He was terminally ill with gross oedema and was seen by Sir Frederic Still who was the only physician in England who confined his work to children. He recommended warmth to induce sweating and a lot of milk was prescribed but the prognosis he gave to the parents was very gloomy. The child continued to deteriorate and on his second visit he asked the child what he wanted to do when he grew up. "I want to be a children's doctor like you Sir" the child answered. He was then seen by a few other physicians and finally by a renal physician who said it was only a matter of time. Suddenly, after a stormy course, his nephrosis cleared following an attack of measles. Fifteen years later the scene shifts to the Membership Exam of the Royal College of Physicians. The boy is now a candidate and Sir Frederic Still was the examiner. It did not take much time for Sir Still to realise that this was the boy to whom he gave a gloomy prognosis many years ago. The candidate sailed through the exam with great ease, and went on to become a distinguished paediatrician and later a Professor of Paediatrics and the President of the Royal College of Physicians.

Today, a disturbance in the Th1 and Th2 immune mechanisms, mediated by cytokines and

${ }^{1}$ Senior Lecturer in Paediatrics, Faculty of Medicine, University of Peradeniya lymphokines, are thought to be involved in the genesis of NS. These observations represent the scientific rationale for the use of cytotoxic and immunosuppressive drugs in childhood NS. With the introduction of antibiotics, and later with the use of corticosteroid therapy, the mortality has fallen dramatically.

Prednisolone was first prescribed for children with NS in 1956, when Arneil described four children, who responded to prednisolone at a dose of $60 \mathrm{mg}$ daily $^{2}$. Although a majority of children will respond to corticosteroid therapy, this therapy is not curative, and over $70 \%$ of children subsequently relapse. This results in the administration of further courses of high dose corticosteroids placing the child at risk of many steroid related side-effects.

In 1967 cyclophosphamide (CYC) was reported as an effective agent in maintaining sustained remission in steroid sensitive nephrotic syndrome (SSNS). The side effect profile of CYC has again been a cause for concern viz. alopecia, bone marrow suppression leading to opportunistic infections, haemorrhagic cystitis and an unquantified long-term risk of infertility and malignancy with obvious adverse implications for children.

In the mid 1980's the immunomodulatory drug levamisole (LEV) and calcineurin inhibitor cyclosporin A (CSA) were added to the armamentarium of therapies with variable success rates and side effects. While the incidence of reported side effects with LEV therapy is low, the side effect profile of CSA such as hirsutism, gingival hypertrophy and potential nephrotoxicity have limited its use in $\mathrm{SSNS}^{3}$.

In contrast, steroid resistant NS is a more heterogeneous group, majority of which have focal segmental glomerulosclerosis (FSGS) on histology. In FSGS the natural history is relentless progression to end stage renal failure and the response to cytotoxic therapy is unpredictable. 
My interest in childhood NS began in 1992 when I started my paediatric career as a Registrar in Paediatrics at the Teaching Hospital Peradeniya. The research work I did in Sri Lanka helped me to secure a post in the Paediatric Nephrology Unit at the Great Ormond Street Children's Hospital, United Kingdom (UK) where I received my training in paediatric nephrology. Since my return to Sri Lanka in 2002 I have established paediatric nephrology services at the Teaching Hospital Peradeniya to which patients are referred from all parts of the country for specialised treatment. Today I shall present to you the research I have conducted both in Sri Lanka and in the United Kingdom in investigating many facets of NS which still remain controversial. These are;

1. Treatment of the initial episode 2. Prevention of relapses
3. Steroid sparing therapy

4. Treatment of steroid resistant disease

5. Long-term outcome

\section{Treatment of the initial episode}

In spite of many years of intensive investigation and review, there is no uniform agreement amongst paediatricians on the ideal treatment protocol for the initial episode. I therefore presented a project proposal to the British Association of Paediatric Nephrology at their Annual Research Meeting in November 2000 in London, to compare the outcome of a prolonged prednisolone regimen with the standard regimen in the context of a randomised placebo controlled trial. This chart contrasts the difference in tapering of prednisolone in the two treatment protocols.

\section{Comparison of 2 Regimens}

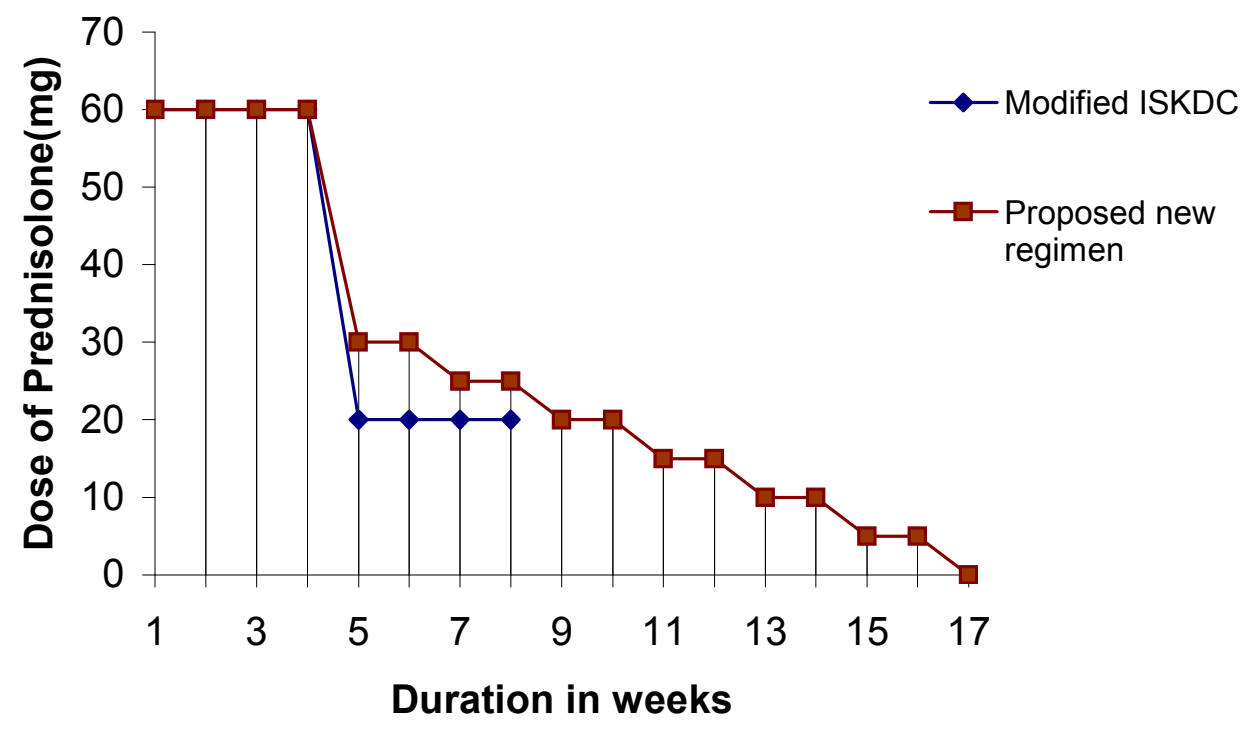

This proposal received the support of 12 out of 13 Paediatric Nephrology Centres in the UK. I battled for one year with the Multi Centre Research Ethics Committee to obtain ethical approval for a national multicentre trial in the UK. The trial was meticulously designed to overcome the shortcomings of other trials in published literature. The pilot trial is now complete and the multicentre study is about to begin in the UK.

\section{Prevention of relapses}

A majority of children with SSNS run a relapsing course. Relapse is associated with the increased risk of complications such as hypovolaemia, thrombosis and infection.

\section{Relapse of NS following MCCV}

Two months after the launch of the national meningococcal $\mathrm{C}$ conjugate vaccine (MCCV) programme in 1999, a child presented with a relapse of NS claimed to be following the vaccination. At this point we reassured the family that it could be a coincidence. Two weeks later another child presented with a relapse of NS following the vaccination. To explore a causal relationship between the relapse and administration of $\mathrm{MCCV}$, the relapse rate during the 12 months pre and post vaccination period were studied. From a population of 224 patients who attended the NS outpatient clinic, 106 patients received the MCCV during first 12 months of the campaign. The occurrence of relapses 12 months before and after 
MCCV was recorded. Comparison of the number of relapses in the 12 months pre and post vaccination periods clearly indicated more relapses during the 12 month post vaccination period. Overall 63 relapses occurred 12 months prior to vaccination compared to 96 during the 12 month post vaccination period. In the 6 months post vaccination period the fraction of relapses attributable to vaccination was 26 . Therefore, out of 106 doses of vaccine, 26 relapses were attributable to the vaccine, which is a risk of 1 relapse for every 4 doses given to this population. This is the first report of a series of patients where a relapse of NS was noted to cluster following an administration of a vaccine.

Conjugate vaccines work by recruiting $\mathrm{T}$ cell help and the cell derived cytokines to aid $B$ cell antibody production and generate memory cells. As the cytokines may play a critical role in the pathogenesis of NS, the disturbance of the cytokine milieu by the MCCV may have resulted in the observed cluster of relapses.

These results suggest a causal relationship between the administration of MCCV and relapse of NS. There could be other immunisations which are capable of triggering a relapse of NS and therefore the paediatricians should keep close vigil on children during immunisation ${ }^{4}$.

\section{Adrenocortical suppression and relapse of nephrotic syndrome}

Baby $\mathrm{H}$ was $1 \frac{1}{2}$ years when he developed NS. His elder sister too had steroid dependent nephrotic syndrome (SDNS) and was treated with many cytotoxic agents. As a result of SDNS he was treated with LEV at $2 \frac{1}{2}$ years and with $\mathrm{CYC}$ at 3 years. At 4 years he was still steroid dependant receiving alternate day prednisolone. His pattern of proteinuria was rather unusual with many spontaneous remissions. When I expressed my surprise the father confessed to me that he was administering daily prednisolone instead of alternate day prednisolone during the periods of proteinuria thus explaining the reason for spontaneous remissions. This prompted me to undertake a comprehensive literature review on adrenocortical suppression and NS. To my surprise there were many unanswered questions with regard to alternate day prednisolone therapy and adrenal suppression.

- Does adrenal suppression occur with long-term alternate day prednisolone? If so

- What is the critical dose at which adrenal suppression is likely to occur?
- Is there an impact created by concurrent administration of levamisole or cyclosporin A with prednisolone on adrenal suppression?

- Does adrenal suppression increase the risk of relapse with NS?

We used the modified synacthen test to evaluate the adrenal axis. A cortisol response $>500 \mathrm{ng} / \mathrm{l}$ was considered normal. Ethical approval for this study was obtained from the Institute of Child Health, University College, London, UK. Thirty two patients underwent evaluation, 12 on alternate day prednisolone, 11 on alternate prednisolone + levamisole and 9 on alternate prednisolone + cyclosporin A. There was no significant difference in the mean dose of prednisolone administered to the 3 groups.

\section{Results}

A significant proportion of children had a peak serum cortisol response below 500ng/l indicating adrenal suppression. In the 2 groups, pred and pred $+\mathrm{LEV}$, over $70 \%$ had evidence of adrenal suppression. Interestingly, in spite of receiving the longest duration of prednisolone, the CSA group had only $25 \%$ of patients with evidence of adrenal suppression. The $\mathrm{P}$ value of 0.01 was highly significant. During a 3 year follow up, 20 children who had a suboptimal cortisol response had a significantly greater number of relapses when compared to the 12 children with a normal cortisol response $(\mathrm{p}=0.01)$.

Our data indicate that $>60 \%(20 / 32)$ of children receiving alternate day prednisolone for steroid dependant NS had a suboptimal cortisol response suggesting adrenal suppression. Therefore this highlights the importance of evaluating adrenal function in children receiving alternate day prednisolone at regular intervals. The results of this study also confirm that children with adrenal suppression are at a greater risk of relapse than those with a normal adrenal function following glucocorticoid therapy. Children with evidence of adrenal suppression should therefore be considered for steroid sparing therapy in order to allow the adrenal axis to recover ${ }^{5}$.

In order to substantiate the findings of this study a further study was undertaken to explore the effects of increasing the dose of prednisolone during viral upper respiratory tract infections to reduce the risk of relapse in children with SDNS. The study was designed to test the hypothesis that a small and short term increase in the dose of prednisolone during viral infections would reduce cytokine release and thereby reduce the risk of relapse in NS. Ethical approval for this study was obtained from the Institute of Child Health, University 
College, London, UK. In each patient the occurrence of relapse of NS was recorded for two consecutive viral upper respiratory tract infections (URTIs). At the first sign of a presumed URTI, parents were asked to report to the principal investigator for a clinical examination. If the predetermined criteria to diagnose a viral URTI were met, these children were randomly allocated to take medicine $\mathrm{A}$ or $\mathrm{B}$ containing either prednisolone or placebo with the first viral URTI and vice versa with the second. There was a significant reduction in the number of relapses when the dose of prednisolone was increased during a viral URTI as the 40 episodes treated with a placebo were followed by $19(47.5 \%)$ relapses while there were only $7(17.5 \%)$ relapses in the prednisolone treated group $(p=0.003)$. The results of this study confirmed the hypothesis that increasing the dose of prednisolone during viral URTIs is capable of reducing the risk of relapse in NS by $30 \%$ and thereby reducing the need for cytotoxic therapy with its associated side-effects ${ }^{6}$.

\section{Steroid sparing therapy}

In spite of many years of intensive investigations and review a rational treatment protocol still remains elusive partly because of the relatively low prevalence of the disease which makes it difficult to record the natural history in an adequate number of patients. A retrospective review was therefore undertaken to establish the therapeutic strategies, complications and outcome of children with NS referred to a tertiary centre over a twenty year period. The Great Ormond Street Hospital (GOSH) is a tertiary referral centre serving a population of approximately six million. The Renal Unit at GOSH runs a special clinic for children with NS. The case notes of all children who received steroid sparing agents for NS from 1980 to 2000 were reviewed.

From 1980 to 2000,863 children with NS were referred to GOSH. There were 610 children with SSNS and 253 with steroid resistant nephrotic syndrome (SRNS). Out of 610 children with SSNS 539 satisfied the entry criteria. Two hundred and eighty $(51.9 \%)$ children received one or more courses of steroid sparing therapy. CYC was the preferred first steroid sparing therapy prescribed in $178(72 \%)$ children. Life table analysis revealed that $53 \%$ and $32 \%$ of children following a course of $\mathrm{CYC}$ remained in sustained remission at one year and five years respectively. LEV was prescribed for 121 children and in 65 it was the first course of steroid sparing therapy. Side effects were rarely encountered during LEV therapy. CSA was used in 81 children in order to control the disease and in 9 pubertal children it was prescribed as the first steroid sparing therapy. Chlorambucil was used in
16 children when other forms of steroid sparing therapy had failed ${ }^{7}$.

Analysis of children with SSNS presenting to Teaching Hospital Peradeniya from 1992-2004 and a comparison with the UK cohort

This study was carried out at the Teaching Hospital Peradeniya (THP), a tertiary referral centre for the central province of Sri Lanka. Outcome of patients admitted to THP with NS between 1992 and 2004 was reviewed. Treatment strategies were similar to the United Kingdom apart from a few exceptions. Chlorambucil, azathioprine and CSA were not prescribed for this cohort of patients. From 19922004, 226 patients were treated for NS of which 197 were SSNS and 29 were SRNS. Seventy seven children received steroid sparing therapy. LEV was the preferred first steroid sparing therapy prescribed for 61 children. CYC was used in 37 patients. Infection is a universal concern in children with NS especially when receiving cytotoxic or immunosuppressive therapy. In the cohort of patients from the UK with SSNS septicaemia and peritonitis were rare complications and none of the deaths were related to infection. However, the incidence of serious infections was 5 fold in Sri Lanka when compared to the UK and 1 death was related to an infection during a relapse of NS. Three children from the UK cohort died during the follow up period, the first from venous thrombosis following diuretic therapy, the second from acute pulmonary oedema following an albumin infusion and the third from acute haemorrhage from a duodenal ulcer during corticosteroid therapy for the relapse of NS. In the Sri Lankan cohort two patients died during follow up. One death was related to cerebral vein thrombosis following hypovolaemic shock while the other death was related to gram negative septicaemia. Levamisole was the mainstay in steroid sparing therapy in Sri Lanka while in the UK it was cyclophosphamide.

Mortality from SSNS is rare. Infections remain a major cause of morbidity and mortality in the developing world but are rare in the Western countries. Hypovolaemia and thrombosis however continue to cause morbidity and mortality in the developing world as well as in the $\mathrm{West}^{8}$.

Efficacy of levamisole as a single agent in maintaining remission in SDNS

Levamisole (LEV) was originally developed as an anthelmintic drug and was subsequently shown to have immunomodulatory properties. This randomised controlled study was carried out between 2002 and 2005 at THP to evaluate the efficacy of LEV as a single agent in maintaining remission in SDNS. Sequential children who had completed two years of therapy with LEV and prednisolone were randomised into 2 groups. In the 
control group all medications were stopped and in the test group levamisole was continued as a single agent on an alternate day basis for a period of one year. The relapse rate was compared at the end of one year in both groups. Seventy six patients were enrolled into the study. There were 34 in the control group and 42 in the test group. At the end of one year of follow up $76 \%$ in the control group suffered a relapse while only $19 \%$ suffered a relapse in the test group $(\mathrm{p}<0.001)$. No significant side effects were noted. The results of this study demonstrate that LEV is safe, easily affordable and effective as a single agent in maintaining remission in SDNS. This can reduce the repeated administration of high dose prednisolone and the use of other cytotoxic agents. The major advantage of LEV is its steroid sparing effect with minimal toxicity ${ }^{9}$.

\section{Steroid resistant nephrotic syndrome (SRNS)}

A majority of children with SRNS have FSGS. This entity was first described in 1957 by Rich as "a hitherto undescribed vulnerability of juxtamedullary glomeruli in lipoid nephrosis". Although FSGS is generally believed to carry a poor prognosis in refractory nephrotic patients, the favourable long-term renal survival found in children who enter sustained remission has led to the emergence of more aggressive immunosuppressive regimens. However, currently there are no universally accepted clinical criteria or histological features that predict a favourable response to immunosuppressive therapy

The aim of this study was to analyse the treatment protocol and the long term outcome of children with primary idiopathic steroid resistant FSGS. The study also focused on clinical, pathological, and immunohistochemical features at presentation that might help to identify children who would have a favourable response to cytotoxic therapy. From a population of 863 patients with NS referred to the Great Ormond Street Children's Hospital, London in the UK from 1980 to 2000,153 children had FSGS as their initial glomerular lesion and 91 had a well documented follow-up for 10 years. The review of histolopathological features was performed by two independent paediatric renal histopathologists who were blinded to the clinical details and outcome of these patients. Based upon histological review, a further 25 patients were excluded for suspected other aetiologies and a total of 66 was identified as having 'pure' idiopathic FSGS. In 51 children cyclophosphamide was prescribed as the first cytotoxic agent while 15 received cyclosporine $\mathrm{A}$ and complete remission was induced in $43 \%$ and $40 \%$ of children respectively. Of the 35 patients who entered complete and stable remission the renal survival was over $90 \%$ while in the 31 non-responders it was $48 \%$ at 10 years.

The multivariate analysis using unconditional logistic regression method identified the presence of mesangial expansion $(p=0.011)$ and tip lesions $(p=0.005)$ as the independent predictors of favourable response to cytotoxic therapy and the presence of renal impairment $(\mathrm{p}=0.008)$ and extensive focal segmental sclerosis $(p=0.025)$ as independent predictors of unfavourable response ${ }^{10}$.

\section{SRNS (Sri Lanka)}

In the Sri Lankan cohort there were 29 children with SRNS of whom 16 had FSGS, 10 MCD and 3 had membrano-proliferative glomerulonephritis. CYC was used to induce remission in FSGS and in MCD. However therapeutic options were limited for children who were CYC resistant. In the developed countries these children are treated with CSA, which is extremely expensive and not freely available in Sri Lanka.

Manusha developed NS at the age of $1 \frac{1}{2}$ years. His elder sister died due to steroid and cyclophosphamide resistant NS. His NS too was resistant to steroids and cyclophosphamide therapy. When he came to THP he was 2 years and was bed bound due intractable oedema and severe steroid toxicity. As cyclosporine A was not then available for us he was treated with pulsed vincristine after discussing with parents as this was the first documented child in Sri Lanka to receive vincristine for NS. He surprisingly entered complete remission in the sixth week but needed a lot of physiotherapy to make him mobile due to steroid induced myopathy. He is now 9 years and is in complete remission and off all medications.

\section{Pulsed vincristine therapy in SRNS}

In order to determine the efficacy of vincristine therapy in steroid and cyclophosphamide resistant nephrotic syndrome 14 patients were treated with weekly pulses of vincristine $1.5 \mathrm{mg} / \mathrm{m}^{2}$ for 8 weeks. Corticosteroid therapy was tapered over 12 weeks. There were 8 males and 6 females of whom 4 had FSGS and 10 had minimal change disease (MCD). Complete remission was induced in $64 \%$ of patients. Of those $44 \%$ maintained sustained remission at 24 months. Notable side-effects were alopecia in $85 \%$, constipation and abdominal distension in $21 \%$, local irritation due to extravasation in $21 \%$ and reversible bilateral ptosis in $14 \%$ patients. Bone marrow suppression and serious infections were not observed in this cohort of patients. The results of this study indicate that vincristine is a cheap, effective and safe agent in inducing and maintaining remission in steroid resistant NS due to FSGS as well as in MCD. It can 
therefore be an attractive alternative in countries where cyclosporine A is not freely available ${ }^{11}$.

Even in the $21^{\text {st }}$ Century the molecular mechanism involved in the generation of NS remains a mystery. Our therapeutic approaches have therefore been based on clinical observations rather than on a scientific hypothesis. Hence ideal protocols to treat NS remain elusive. Moreover, the data suggests that the disease expression and outcome of NS in different countries could be variable. Hence there is a need to organise clinical trials in Sri Lanka to provide robust evidence in order to develop ideal treatment protocols for Sri Lankan children. I therefore hope that my presentation today will generate an enthusiasm amongst the members of the SLCP to conduct such trials. Cytotoxics and immunosuppressive drugs undoubtedly play an important role in the treatment of childhood NS, although these drugs by themselves are associated with significant morbidity and even mortality. The benefits of immunosuppressive therapy in SRNS should be meticulously balanced against toxicity keeping in mind that refractory NS only results in renal death, and renal transplantation has a good long-term outcome in these patients.

The famous rugby star Jonah Lomu stormed into the rugby world in 1995 when he ran through the entire English defence to score 4 tries bringing victory to All Blacks. He was virtually unstoppable. But he was stopped by SRNS. However he almost made a great comeback to the rugby scene after a successful renal transplant and became a role model for children with NS. Until recently in Sri Lanka when children progress to ESRF we had to painfully watch them dying as we were not able to offer them renal transplantation.

When the second Medical School was started, Prof $\mathrm{CC}$ de Silva took up the challenge of organizing the Department of Paediatrics in Peradenya in 1964. $\mathrm{He}$ then continued to make weekly visits to Peradeniya until the department got going. I am sure we have done Professor CC de Silva proud as it was the Department of Paediatrics, University of Peradeniya that was instrumental in starting the first Paediatric Renal Transplantion Programme in Sri Lanka. Due to a superb team effort by the Departments of Paediatrics, Surgery and Anaesthesiology, with the help of the Nephrology Unit in Kandy and a renal transplant surgeon from the UK, the much needed Paediatric Renal Transplant Programme was commenced at the THP in 2004. But unfortunately, after 7 successful transplants were performed within 3 months, the entire programme came to a grinding halt due to certain differences in opinion that surfaced and the life of a child who was awaiting a renal transplant was in great danger because we did not have a paediatric dialysis programme at that time. However, there was an overwhelming response from the public and professionals that this life saving humanitarian service should continue. As a result of successful intervention and negotiation by several important persons, the Paediatric Transplantation Programme recommenced but it was a day too late to the save the life of the unfortunate child whose parents really battled hard to reactivate this programme. However today we have our own transplant surgeon and 36 living related transplants have been performed with over $95 \%$ graft and patient survival rate over a 5 year period which is comparable to that of any advanced Western unit aptly justifying the decision to recommence this programme ${ }^{12}$. President Sir, Ladies and Gentlemen, let us provide our children with kidney disease a normal life.

\section{References}

1. Anonymous. A notable case of nephrosis. Arch Dis Child 1978; 53:363-5.

2. Arneil GC. Treatment of nephrosis with prednisolone. The Lancet 1956; 14:409-10.

3. Abeyagunawardena A, Brogan PA, Trompeter RS, Dillon MJ. Immunosuppressive therapy of childhood idiopathic nephrotic syndrome. Expert Opinion on Pharmacotherapy 2002; 3(5):513-9.

4. Abeyagunawardena A, Goldblatt D, Andrews N, Trompeter RS. Risk of relapse after meningococcal $\mathrm{C}$ conjugate vaccine in nephrotic syndrome. Lancet 2003; 362:449-50.

5. Abeyagunawardena AS, Hindmarsh $P$, Trompeter RS, Adrenocortical suppression increases the risk of relapse in nephrotic syndrome. Arch Dis Child 2007; 92(7):585-8.

6. Abeyagunawardena AS, Trompeter RS. Increasing the dose of prednisolone during viral infections reduces the risk of relapse in nephrotic syndrome: a randomised controlled trial. Arch Dis Child. 2008; 93(3):226-8.

7. Abeyagunawardena A, Dillon MJ, Rees L, van Hoff W, Trompeter RS. The use of steroid sparing agents in steroid sensitive nephrotic syndrome. Pediatric Nephrology 2003; 18: 919-24.

8. Abeygunawardena A. Nephrotic syndrome in the 3rd world: causes, approaches, outcomes, comparisons with the western practice: Pediatric Nephrology 2004; 19:C48. 
9. Abeyagunawardena AS., Trompeter RS, Efficacy of Levamisole as a single agent in maintaining remission in steroid dependant nephrotic syndrome. Pediatric Nephrology 2006; 21:1503.

10. Abeyagunawardena AS, Sabire NJ, Risdon T, Kumarasiri PVL, Dillon MJ, Rees L, van Hoff $\mathrm{W}$, Trompeter RS. Predictors of long term outcome of children with idiopathic focal and segmental glomerulosclerosis. Pediatric Nephrology 2007; 22(2):215-21.
11. Abeygunawardena A, Abeysekera C, Thalgahagoda S, Dillon MJ. Pulsed vincristine therapy in steroid resistant nephrotic syndrome: Pediatric Nephrology 2004; 19:C66.

12. Abeysekera CK, Gunasekara WD, Abeygunawardena A, Buthpitiya AG, Lamawansa MD, Fernando O, Goonasekera CD. First experiences of paediatric kidney transplantation in Sri Lanka. Pediatr Transplant. 2007;11(4):408-13 